mê pæt wyrd zewæf and zewyrht forzeaf pæt ic zrôfe zræf and pæt zrimme zeræf fleón flâsce ne mæz.

Dass in dem ersten verse forzaf zu lesen ist, habe ich Beitr. IX, 235 bemerkt. Ausserdem nehme ich anstoss an Grein's conjectur zercef für handschriftliches zrref. Das wort ist unerklärt, Grein selbst kann nur zweifelnd als bedeutung desselben 'constitutum, destinatum' vermuten. Ohne zweifel ist zrof in $71^{\mathrm{b}}$ nur widerholung des $z$ ref von $71^{\mathrm{a}}$, man braucht sich also nicht zu ängstlich an seine buchstaben $\mathrm{zu}$ halten. Mit scraf wird dem verse wie dem sinne vollkommen gentigt. TÜBINGEN, 18. august 1885 .

\title{
DIE HEIMAT DES BEOWULFDICHTERS.
}

In diesen Beiträgen XI, $173 \mathrm{ff}$. sucht $\mathrm{G}$. Sarrazin seine annahme eines nordischen ur-Beowulf in liedform unter anderem auch durch eine reihe von sprachlichen argumenten zu stlutzen. Ich möchte diesen versuch nicht obne einsprache bingehen lassen; es könnte sonst doch am ende jemand auf den wahn verfallen, als verhielte es sich mit den besprochenen dingen wirklich so wie der verfasser jenes aufsatzes seine leser glauben machen will. Dabei lebne ich es ausdrucklich ab, auf discutable punkte einzugehen, d. h. auf dinge, wo meinung gegen meinung stehen kann. Meine kritik soll sich bloss auf dinge erstrecken, welche Sarrazin als angeblich factische grundlagen seiner auffassungen benutzt. Vollständigkeit in der widerlegung des widerlegbaren habe ich dabei nicht im auge.

Seine beweisfuhrung hebt Sarrazin s. 173 mit der behauptung an, der Beowulf wie die tubrige ags. dichtung zeige altnordischen einfluss im wortschatz und in der phraseologie. Diese behauptung wird dann weiter gestutzt auf eine liste von 36 wörtern, die dem Beokulf entnommen sind, aber auch sonst in der ags. poesie begegnen, dagegren der ags. prosa fehlen. Diese 36 poetischen wörter werden danach fur scandinavische fremdwörter erklärt. Sehen wir uns dieselben also etwas näher an! 
Ags. brezo, breozo soll aus altn. bragr entlehnt sein. Ich wäre begierig zu wissen, wie die Angelsachsen aus der form bragr oder meinetwegen urnordisch *bragiR oder *bragiz die form brezu gemacht haben sollen. Ausserdem passt die bedeutung nicht, denn ags. brezu bedeutet schlechthin 'herscher, beherscher', altn. bragr aber den 'besten, trefflichsten'. Alte wurzelverwantschaft der beiden wörter wird damit nicht geleugnet (Grimm, Myth. 194 f.; beachte auch Brezowine npr. Chr. a. 759$)$.

freca $=$ altn. freki. Die identität beider wörter ist unzweifelhaft, denn beide sind schwache substantiva zu dem gemeingerm. adj. got. -friks, altn. frekr, ags. frec, ahd. freh. Áber altn. freki beảeutet 'wolf'; dass das ags. freca, welches zur bezeichnung von helden dient, jemals auch diesen sinn gehabt und nicht direkt 'der kuhne, kampfgierige' bedeutet habe, wird zwar z. b. von Grein I, 338 angenommen, ist aber absolut unerweislich. Auch mhd. heisst vrech noch sehr gewöhnlich 'klihn', s. z. b. Mhd. wb. III, 396.

beorn = altn. bjorn. Ich vèrmute, dass Sarrazin nicht daran gedacht hat, dass ags. beorn 'held, mann, mensch', aber altn. bjorn 'bär' heisst. Die zahlreichen auch deutschen eigennamen, die mit bern gebildet sind, wird ja auch wol Sarrazin nicht aus dem norden stammen lassen.

$\sec z=$ altn. seggr. Eine unzweifelhaft richtige gleichung. Aber auch im Heliand steht das wort fünfmal. Ist denn auch der Heliand aus dem nordischen ubersetzt?

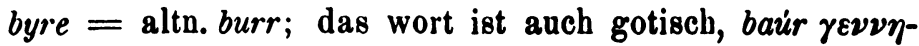

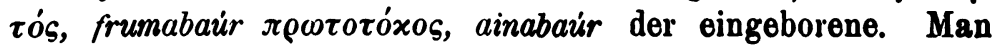
sieht also nicht, weshalb das wort aus dem nord. entlehnt sein muss. Das gleiche gilt von

mazo = altn. mogr. Hier haben wir nicht nur got. magus, sondern auch alts. magu nebst dem compositum magujung, und ahd. mayazogo, magazoha.

byrle = altn. byrlari, byrli. Die flexion stimmt nicht. Ausserdem fehlt das wort keineswegs der ags. prosa. Bereits Grein I, 152 gibt glossenbelege dafur; weiteres s. bei BosworthToller s. v. byrle.

serce = altn. serkr, ersteres swf., letzteres stm.! Auch ist ist ags. serce der prosa keineswegs fremd: serce armilausia 
Wright-Wulcker 7, 5. 349, 35, vgl. serc colobium Hpt. gl. $493^{\mathrm{b}}$ (letzteres bei Leo citiert), syrc colobium Wright-Wulcker 125, 1, suppar, interula ib. 187, 17.

heoru $=$ altn. hjorr. Dabei ist sowol das got. hairus wie alts. heru- in den compositis heru-bendi, -drôrig, -grim, -sêl, -thrum ausser acht gelassen. Von diesen compositis begegnen sich einige geradezu mit angelsächsischen, vgl. heorudreóriz, heoruzrim.

darod = altn. darraðtr. Dann ist wol auch ahd. tarl lancea, sagitta (Graff V, $457=$ Ahd. gl. II, 463, 52. 546, 60) aus dem nordischen entlehnt, auch franz. dard, ital. span. dardo?

rês $=$ altn. rás. Die prosabelege gibt schon Lye. Dazu kommt das verbum rếsan mit seinen compositis, wie berêsan, onrêsan, ebenfalls in prosa nicht selten; vgl. z. b. schon rêssde inruit in den Corpusglossen, Wright-Wulcker 28,1, onrêsa Vesp. Ps. 61, 4, onrếsdun ib. 58, 4, berês ठ Cura past. 143, 6, berâsað ib. 295, 20 etc. etc.

orleze $=$ altn. orlog. Das erstere wort ist durch alts. urlagi, orlag, abd. urlag vor jedem verdacht einer entlehnung geschutzt. Es ist gewiss ein alter os-es-stamm.

wicz = altn. viggr (!) oder vielmehr vigg, da das wort auch im nordischen neutrum ist. Das wort begegnet auch in Heliand 389.

blanca $=$ altn. blakkr 'ross'. Dass man auch ausserhalb des nordens einen schimmel als 'blankes' pferd bezeichnete, lehrt ein blick auf Graff III, 254, welche stelle bereits von Grein .s. v. blanca angezogen ist.

ben $=$ altn. ben; ubersehen ist das alts. ben(i)wunda.

haf = altn. haf, allen nördlichen seevölkern gemeinsam, wie ein blick auf Schade 2372 lehren kann. Namentlich friesisch ist hef ganz geläufig, Richthofen 803.

sund = altn. sund ist auch prosaisch: mid sunde $p \hat{a} e \dot{a}$ oferfaran Oros. 72, 29 Sweet ist schon bei Lye zu finden, desgleichen gibt Lye belege fur die composita sundzierd, linne, -mere. Weiteres dazu s. bei Wright-Wülcker II, 377. Md. sunt m. (gegen aitn.) s. bei Lexer III, 1319.

bearu $=$ altn. borr ist in ags. prosa ganz gewöhnlich. Ausser den belegen bei Bosworth-Toller 73, Wright-Wulcker 
II, 253 vgl. z. b. noch Cura past. 355, 5. Ep. Alex. 515. 619. 632. 641. 672 Baskerv. Leechd. I, 106. Auch in ortsnamen begegnet das wort, vgl. z. b. sübbeara C. D. 6, 181. 182, ciddesbeara C. D. 6, 182 etc.

leomu 'äste' = altn. limar. Wie erklårt Sarrazin bei seiner annahme einer entlehnung die verschiedenheit des geschlechts? In ags. scheint leomu 'äste' doch nur als plurale tantum vorzukommen, also kann der nordische neutrale sing. lim nicht wol herangezogen werden.

ful 'becher' = altn. full. Alts. ful steht im Heliand.

mene = altn. men; aber auch alts. halsmeni im Heliand, ahd. menni, Graff II, 798. Ausserdem ist das wort in ags. prosa ganz gewöhnlich. Glossenbelege 8. z. b. bei Wright-Wulcker II, 342 s. v. mene, menas und II, 346 s. v. myne (zum teil).

dôzor = altn. dacgr. Das wort kann des fehlenden umlauts wegen nicht entlehnt sein; es ist alter os-es-stamm.

zealdor $=$ altn. galdr ist auch in prosa gebrăuchlich, vgl. z. b. die belege bei Bosworth-Toller und bei Wright-Wulcker II, 294.

snotor = altn. snotr; vgl. aber auch got. snutrs, snutrei, ahd. snottar Graff VI, 845. Reichliche ags. prosabelege s. bei Lye.

frôd $=$ altn. fróor! Bekanntlich ein gemeingermanisches wort, got. frôbs, alts. altfries. frôd, ahd. fruot!

zamol = altn. gamall. Uebersehen ist alts. gigamalôd.

zimfast,$=$ altn. gimfastr. Das sieht auf den ersten blick sehr bestechend aus. Aber erstens ist das einmalige zimfaest im Beowulf nur fehler oder nebenform fur zinfast, zweitens beruht die ansetzung eines altn. gimfastr adj. auf der einzigen höchst zweifelhaften stelle við gimfastan Völundarkv. 5, 4 (Bugge), wo man jetzt mit Bugge wol allgemein við gim fastan liest.

hrinzan = altn. hringja, begegnet auch in ags. prosa, wenn auch verhältnismåssig spăt, s. Bosworth-Toller.

eorclanstân = altn. jarknisteinn. Einen ags. prosabeleg verzeichnet Bosworth-Toller s. 253. Wenn das wort der einen sprache ursprunglich fremd ist, und das ist ja nach der lage der dinge sehr wahrscheinlich, so liegt die entlehnung sicher auf seite des nordischen, wo das wort ganz isoliert steht, 
wăhrend wir auf westgerm. boden sowol das selbstăndige adj. erkan als zahlreiche namen finden die mit diesem adjectiv gebildet sind.

feorhleze (lies-lezu?) = altn. fjorlag kann aus formalen grunden ebensowenig entlehnt sein als orleze aus altn. orlog. Man musste durchaus *feorhlaez erwarten.

Von den 36 angeblichen fremdwörtern sind also sicher einmal $30 \mathrm{zu}$ streichen, weil sie sich entweder als bestandteile auch des prosawortschatzes der Angelsachsen oder als gemeingermanisches eigentum erwiesen haben. Es bleiben dann noch folgende reste:

eodor $=$ altn. jaðarr in der bedeutung 'schutzer, furst'; nur die gemeinschaft der tabertragenen bedeutung kann uberhaupt angezogen werden. Sonst ist das wort ja bekanntlich gemeingermanisch.

missere $=$ altn. misseri. Warum ein wort, das sich auf die altgermanische halbjahrrechnung bezieht, die bei den suldlichen völkern frther durch das christliche jahr abgelöst worden, notwendig aus dem nordischen entlehnt sein muss, sieht man nicht.

facenstafas $=$ altn. feiknstafir. Die bildung von abstractis auf -stafas pl. tant. ist im ags. durchaus gewöhnlich, s. Grein II, 477. Das nord. wort bezeichnet an einer stelle geradezu noch 'verderbliche runen', möglicherweise auch noch an den beiden andern stellen wo es erscheint (8. Egilsson s. v.). Das zusammentreffen beider wörter kann also rein zufällig sein. Dasselbe gilt auch von dem compositum feorhseóc $=$ altn. fjorsjükr.

Als einzige stutzen bleiben hiernach fur Sarrazin ubrig die beiden adjectiva atol und bront $=$ altn. atall und brattr, und die wird man denn getrost auch fur urgermanisch balten durfen.

Nicht minder unglucklich ist Sarrazin in seiner weiteren argumentation. Da soll z. b. pyle s. $174 \mathrm{zu}$ den 'altnordischen' wörtern gehören, welche der Beowulf vor der ubrigen ags. poesie und der prosa voraus hat. Es hat ihm also beliebt die ausfuhrungen Mullenhoff's ther den ags. pyle (Altertumskunde $\mathrm{V}, 1,288 \mathrm{f}$.) und die dort gegebenen prosanachweise zu ignorieren. Auch pyrs soll aus altn. purs entlehnt sein. Wusste 
man nur, wie dann der umlaut sich erklärt! Auch ahd. durs Graff V, 228, existiert nicht fur Sarrazin, um von den eigennamen abermals $\mathrm{zu}$ schweigen.

Ein weiteres beispiel nordischen einflusses im Beowulf findet Sarrazin in der bedeutung der praeposition ymbe Beow. 508. 569. Es soll nämlich hier ymbe in der sonst ungewöhnlichen bedeutung 'über - hin, in, auf' dem altn. um entstammen. Der erste beleg, Beow. $507 \mathrm{f}$. ist naturlich zu streichen, denn ymb sund flite heisst doch nur 'um die schwimmkunst wetteifertest'. Richtig ist das zweite beispiel ymb brontne ford 'auf dem meere'. Hätte aber Sarrazin anch nur z. b. Heyne's glossar zum Heliand nachgeschlagen, so wurde er dort beispiele wio umbi Galileoland 2665, umbi Sodomoland 4370 'tuber Galilaea, das Sodomiterland hin - in Galilaea etc.' gefunden baben. Was dann die heranziehung von wendungen wio altn. fara um ey, fara um sundit anlangt, so will ich der kurze halber annehmen, dass Sarrazin hinlängliche grunde hat, um diese um so ohne weiteres mit sicherheit auf die praeposition umbi zuruckzufubren.

Auch die bemerkung uber furtum Beow. $1707=$ altn. forठum ist ungenau. An der citierten stelle des Beowulf heisst furðum nur 'neulich, jungst': Hroбzar bezieht sich auf die rede, die er nach der tötung Grendels an Beowulf gerichtet hatte, vgl. v. $946 \mathrm{ff}$; altn. forठum aber heisst 'vor alters'. Wo bleibt da die entlehnung?

Aus der 8. 174 noch weiter folgenden langen liste von vergleichungen, die sich meist von selbst richten, hebe ich beispielsweise hervor die bemerkung 8. 175, dass zu beachten sei, dass im altn. tár nicht bloss lacrima, sondern auch gutta heisst. Damit ist gesagt, dass teár im ags. nicht als 'tropfe' vorkomme. Naturlich gilt auch hier wider das genaue gegenteil von dem was Sarrazin behauptet. Grein II, 526 belegt aus der prosa balsames teár, aus der poesie hunizes teár und blôdizum teárum von 'blutstropfen'; andere stellen sind z. b. bei Bosw.-Toller s. v. huniz, hunizteár(lic) zusammengetragen.

S. 176 wird behauptet, die asynthetischen verbindungen wie uncer Grendles kämen in der ags. prosa nicht vor. Beitr. IX, 271 habe ich die prosabeispiele uncer Wulfrices und uncer Brentinzes aus urkunden nachgewiesen. 
S. 177 wird vermutet, der gen. part. in der verbindung hwaet searohobbendra Beow. 237 sei 'wol' nur in der poesie ublich. Wie druckte man denn in der ags. prosa den begriff 'was fur einer' aus? Prosabeispiele s. bei Bosworth-Toller 8. $569 \mathrm{f}$. Und vielleicht uberzeugt sich Sarrazin durch einen blick auf Grimm's gr. IV, $451 \mathrm{f}$. dass diese construction auch altsåchsisch und althochdeutsch ist.

Als 'seltsam und mehr altnord. als altenglischer syntax entsprechend' wird ferner ebenda die construction transitiver rerba mit dem dativ bezeichnet. Voran stehen belege fur onfôn c. dat. mit dem zusatz 'in poesie auch sonst', d. h. also wider 'nicht in prosa'. Nun ist es zwar richtig, dass die ältere ubersetzungsprosa sehr hăufig onfôn mit dem acc. construiert, aber keineswegs ausschliesslich. Die belege für onfôn c. dat. sind so bäufig, dass ich leider nicht besonders dafür gesammelt habe. Doch habe ich zufällig aus Aelfred's Cura past. angemerkt hié onfooð đêre lâre 293, 25, aus dem Beda onfenz hê Jonan wîfe 2,9 p. 131 Wheloc, Jâm zerŷnum onfôn 2,9 p. 132, pâm zeleáfan onfôn 2, 13 p. 141. Der index zu den Blickling Homilies ergibt folgende sichere ${ }^{1}$ ) belege fur den dativ: onfenze hê heora hîwe 29,5, âum hîve onfôn 29,6 , onfêhð eallum pâm zồdum 37,25, phêre mêde ne ne onfồ 41,14, ż̂े onfôn tô lŷtlum leánum 41,21, mannum onfô 49,5, onfenz scinendum wuldorhelme 49,11, onfêhঠ medmycclum feó 61,30, onfô pầe êcan zenyperunza 61,32, heofonlicre mêde onfôn 83,15 , zê onfồ pâm mazne 119,11, onfenzon pâm hâlzan zaste 133,19 , onfenzon pêre mêstan strenze 135, 34, onfôh pissum palmtwize 137,24. 153,14, onfôh pâ mînre sâwle 139,14, onfenzon pdre eádizan Marian 145, 30, onfôn rihtre ondetnesse 155, 1, onfồ mînre mêder 157,32, onfêh hêe ... pếre ... żzaderunze onfenze 165,36 , pếre ... wêtan hiê onfenzon 209,8, him onfenzon pâ nicras 211,5, wêepnum onfôn 213, 2.

Sehr schön ist dann wider s. 182 die zusammenstellung von ags. zerâcan 'erreichen' mit altn. raekja 'curare, expellere,

1) Als sicher habe ich dabei die beispiele für dat. sg. f. mit eingerechnet, weil fur Blickl. die spätws. sebr geläufige construction von onfôn mit dem gen. noch durch kein sicheres beispiel zu belegen ist. 
aversari'. Diese definitionen sind aus Egilsson abgeschrieben, der natülich von einander trennt 1 . rakja = rokja $=$ ags. rêcan, ahd. ruochen, und 2. rakja für *urœekja = gera rækan 'vertreiben'. Nun möchte man wirklich wissen, wie 'erreichen' mit diesen beiden scandinavischen wörtern zusammenkommt!

Ein deutlicher scandinavicismus ist fur Sarrazin die wendung tô bonan weorठan $=$ altn. at bana verða s. 182; dieselbe wendung ist aus dem Hildebrandslied und dem Heliand bokannt, vgl. meine citate, Heliand s. 453. Dass das objekt in den beiden von $S$. hauptsăchlich verglichenen sătzen kein persönliches ist, macht keinen erheblichen unterschied.

Ebenso wenig sind die s. 182 aufgefuhrten constructionen von standa scandinavisch; ich verweise wegen einer Heliandparallele abermals auf meine nachweise im formelverzeichnis 8. 432 unter 'leuchten'.

Den beschluss möge noch eine syntaktische bltte machen. S. 181 scheint die stelle

pæet fram hâm żefræz̆n Hiżelâces pežn

Beow. 194 für Sarrazin besonders beweisend. Fram hâm kann ihm nichts anderes bedeuten als 'ans Seeland', und es ist nach ihm nur zu verstehen, 'wenn man es vom standpunkt des dichters, săngers und seiner zuhörer auffasst'. Der englische bearbeiter behielt 'begreiflicher weise' (!) die wendung bei. Besonders beweisend ist die stelle nur dafur, dass eine wichtige syntaktische regel des ags. Sarrazin unbekannt geblieben ist. Wir heutzutage 'betrachten' uns zwar z. b. ein ding 'in der nähe', aber das ist unanschaulich und unlogisch. In alten zeiten 'sah' man es noch 'aus der năhe oder ferne', von einem naben oder fernen standpunkt aus. So lesen wir z. b. im Beowulf richtig neán in der stelle pat zê zenôze neán sceániat beázas ond brâd zold $3105 \mathrm{f}$. Ebenso 'erwartet' man jemand 'aus der nähe': zif $p \hat{a}$ Grendles dearst nihtlonzne fyrst neìn bidan Beow. $527 \mathrm{f}$. Man hört zwar einen lärm 'weithin', aber der in der ferne befindliche hörer hört ihn doch 'aus der ferne': hlôh ond hlŷdde, hlynede ond dynede, pat mihten fîra bearn feorran zehŷran, hâ sê stîtmôda styrmde ond zylede Judith 23 ff. Man crinnert sich eines jungstvergangnen ereignisses wider 'aus der nähe': wê bas hereveorces, hlêfdize mân, for $n \hat{y} d-$ 
pearfe ned́n myndziad Elene $656 \mathrm{f}$. Auch die sterne die dem nordpol nahe stehen drehen sich 'aus der năhe' um die axe: ôtru tunzl ... forkấm hî̀ pầre eaxe atan ymbhwerfað, pone norðende neán ymbcerrað Metra $28,13 \mathrm{f}$. Ebenso werden aber auch endlich die verba des 'erfahrens, horens' construiert. Zeugnis ist dafur wider der Beowulf: sydðan aðelinzas feorran zefriczean fleam eówerne $2889 \mathrm{f}$. 'wenn fernwohnende helden von eurer flucht hören'. So ist denn auch jenes fram hâm zefroezn fur unsere anschauung nichts anderes als 'es hörte in seiner heimat'.

TÜBINGEN, 22. sept. 1885.

E. SIEVERS. 